\title{
Reversal of autoimmune diabetes by restoration of antigen-specific tolerance using genetically modified Lactococcus lactis in mice
}

\author{
Tatiana Takiishi, ${ }^{1}$ Hannelie Korf, ${ }^{1}$ Tom L. Van Belle, ${ }^{1}$ Sofie Robert, ${ }^{1}$ Fabio A. Grieco, ${ }^{2}$ \\ Silvia Caluwaerts, ${ }^{3}$ Letizia Galleri, ${ }^{2}$ Isabella Spagnuolo, ${ }^{2}$ Lothar Steidler, ${ }^{3}$ \\ Karolien Van Huynegem, ${ }^{3}$ Pieter Demetter, ${ }^{4}$ Clive Wasserfall, ${ }^{5}$ Mark A. Atkinson, ${ }^{5}$ \\ Francesco Dotta, ${ }^{2}$ Pieter Rottiers, ${ }^{3}$ Conny Gysemans, ${ }^{1}$ and Chantal Mathieu ${ }^{1}$ \\ ${ }^{1}$ Clinical and Experimental Endocrinology, KU Leuven, Leuven, Belgium. ${ }^{2}$ Diabetes Unit, Department of Internal Medicine, \\ Endocrine and Metabolic Sciences and Biochemistry, University of Siena and Fondazione Umberto Di Mario ONLUS, \\ Siena, Italy. ${ }^{3}$ ActoGeniX NV, Zwijnaarde, Belgium. ${ }^{4}$ Department of Pathology, Université Libre de Bruxelles (ULB), Brussels, Belgium. \\ ${ }^{5}$ Department of Pathology, Immunology and Laboratory Medicine, College of Medicine, University of Florida, Gainesville, Florida, USA.
}

\begin{abstract}
Current interventions for arresting autoimmune diabetes have yet to strike the balance between sufficient efficacy, minimal side effects, and lack of generalized immunosuppression. Introduction of antigen via the gut represents an appealing method for induction of antigen-specific tolerance. Here, we developed a strategy for tolerance restoration using mucosal delivery in mice of biologically contained Lactococcus lactis genetically modified to secrete the whole proinsulin autoantigen along with the immunomodulatory cytokine IL-10. We show that combination therapy with low-dose systemic anti-CD3 stably reverted diabetes in NOD mice and increased frequencies of local Tregs, which not only accumulated in the pancreatic islets, but also suppressed immune response in an autoantigen-specific way. Cured mice remained responsive to disease-unrelated antigens, which argues against excessive immunosuppression. Application of this therapeutic tool achieved gut mucosal delivery of a diabetes-relevant autoantigen and a biologically active immunomodulatory cytokine, IL-10, and, when combined with a low dose of systemic anti-CD3, was well tolerated and induced autoantigenspecific long-term tolerance, allowing reversal of established autoimmune diabetes. Therefore, we believe this method could be an effective treatment strategy for type 1 diabetes in humans.
\end{abstract}

\section{Introduction}

Type 1 diabetes (T1D) is an autoimmune disease characterized by breach in tolerance toward pancreatic insulin-producing $\beta$ cells $(1,2)$. Interventions aimed at arresting $\beta$ cell destruction once the autoimmune attack has set in, as is the case in antibody-positive or newly diagnosed patients, have not been successful, with the exception of trials using frank immunosuppression such as cyclosporine or high doses of nonspecific immune modulators such as anti-CD3 mAbs. High doses of these Abs reverted disease in mouse models of $\operatorname{T1D}(3,4)$ and preserved C-peptide production in recently diagnosed patients for more than 1 year in two phase II studies (5-7). However, the high doses of anti-CD3 required for efficacy were accompanied by moderate cytokine release-related symptoms at the time of administration and transient Epstein-Barr virus reactivation (8), whereas recent studies such as DEFEND-1 (9) and Protégé (10) demonstrate that low doses of anti-CD3 by themselves do not robustly influence the course of $\beta$ cell destruction in T1D in humans. Post hoc analysis in the recent Protégé trial of patient subgroups treated

Authorship note: Tatiana Takiishi, Hannelie Korf, and Tom L. Van Belle contributed equally to this work. Conny Gysemans and Chantal Mathieu share senior authorship. Conflict of interest: Silvia Caluwaerts, Lothar Steidler, Karolien Van Huynegem, and Pieter Rottiers have financial interests in ActoGeniX NV, including employment and stock options.

Citation for this article: J Clin Invest. 2012;122(5):1717-1725. doi:10.1172/JCI60530 with higher doses, however, clearly confirmed the potential of anti-CD3 to modify T1D (10).

More importantly, the resetting of the immune system should be accompanied by a reprogramming toward tolerance for $\beta$ cell-specific antigens (Ags). In prevention studies in NOD mice, administration of putative $\beta$ cell Ags, via routes that promote tolerance such as the nasopharyngeal and intestinal mucosa or the skin, could prevent disease when administered before onset of autoimmunity $(11,12)$. Administration after onset of autoimmunity was, however, not able to modulate disease progression in mice or humans, as has been demonstrated recently in clinical trials (13). These failures may be related to the choice of Ag, dose, route, frequency, and mode of presentation of the Ag to the immune system, but most probably at that stage of disease the Ag-specific therapy should be combined with immune modulation involving reprogramming of $\mathrm{T}$ lymphocytes (14).

In the gut, humans are in intimate contact with billions of commensal bacteria that do not trigger an inflammatory response in healthy individuals, because specific characteristics of the gut immune system create a context that promotes tolerance rather than immunity toward gut-delivered Ags. Harnessing this phenomenon, we have devised a tool for gut delivery of diabetes-relevant autoAgs, together with immunomodulators. Lactococcus lactis, a common and food-grade commensal bacterium that is non-pathogenic and non-colonizing, was genetically modified (GM) to secrete the autoAg human proinsulin alone or in combination with the 

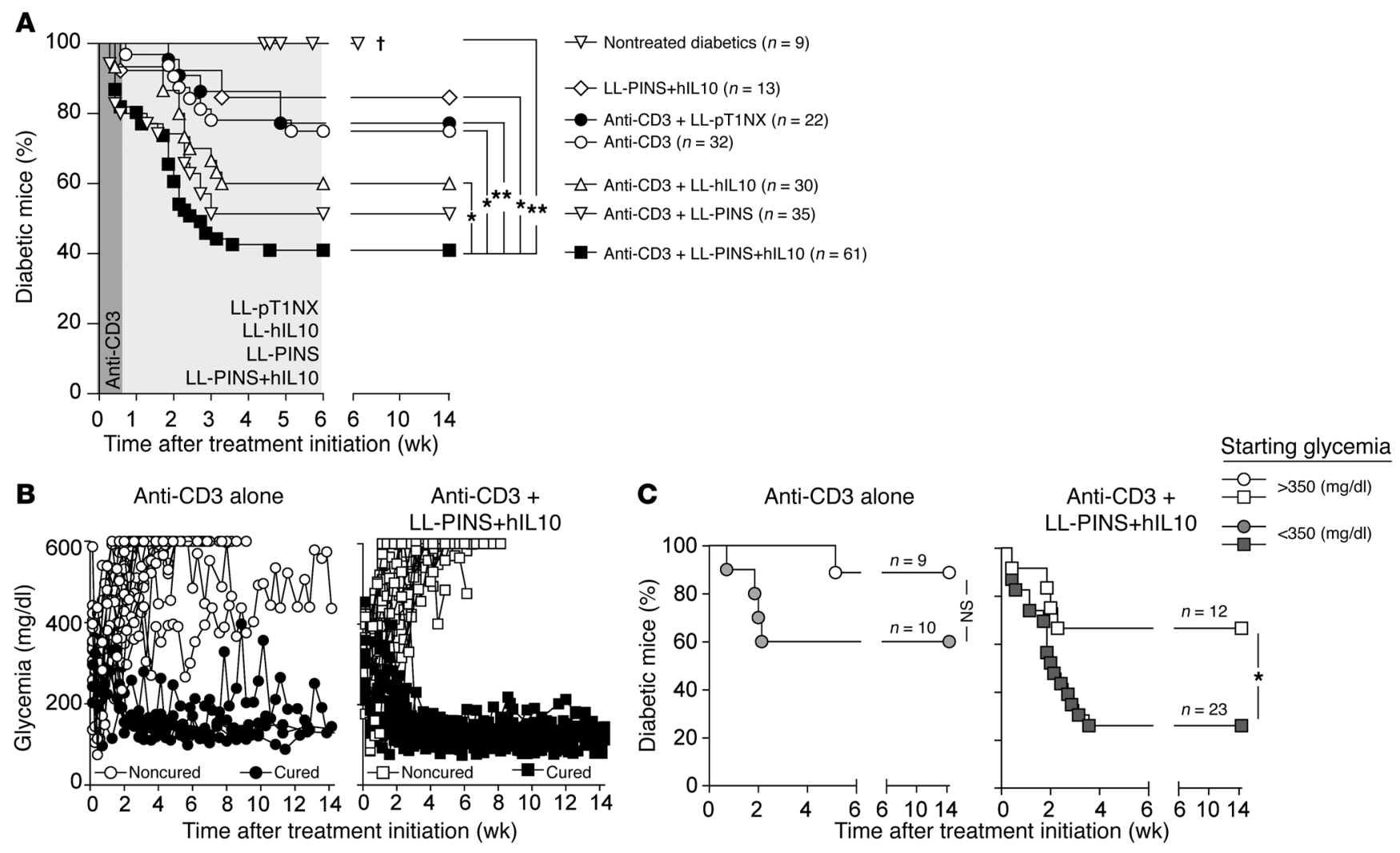

\section{Figure 1}

CT stably reverts autoimmune diabetes in NOD mice. Newly diagnosed diabetic NOD mice were treated as indicated, and glycemia was monitored until 14 weeks after treatment initiation. (A) Percentage of mice that remained diabetic after treatment. $\dagger$ indicates dead or moribund mice. (B) Individual blood glucose levels of newly diagnosed diabetic NOD mice treated with anti-CD3 monotherapy (left panel) or anti-CD3 and LL-PINS+hIL10 CT (right panel). Open symbols, hyperglycemic mice; filled symbols, normoglycemic mice at week 14. (C) Newly diagnosed diabetic NOD mice were stratified based on initial blood glucose level less than (gray symbols) or greater than (open symbols) $350 \mathrm{mg} / \mathrm{dl}$. Shown is the percentage of mice that remained diabetic upon treatment with anti-CD3 monotherapy (left panel) or anti-CD3 and LL-PINS+hIL10 CT (right panel). In all panels, statistical significance between groups was determined by Mantel-Cox log-rank test; ${ }^{*} P<0.05,{ }^{\star \star} P<0.01$.

tolerance-promoting cytokine IL-10. Similar live, biologically contained GM L. lactis bacteria have been applied in other diseases and are being evaluated currently in patients with mucositis (15-20).

Here we demonstrate that, in the context of a short course of low-dose anti-CD3, GM L. lactis secreting human pro-insulin and IL-10 can stably revert autoimmune diabetes in newly diagnosed diabetic NOD mice. Mechanistically the treatment induced Agspecific Foxp $3^{+}$Tregs that prevented diabetes transfer and homed to the islets of Langerhans. These results demonstrate for the first time to our knowledge the potential to arrest T1D by induction of Ag-specific tolerance using a novel, safe tool for gut-delivered $\beta$ cell Ags and biologically active immunomodulators in the context of clinically acceptable low doses of anti-CD3 mAbs.

\section{Results}

Secretion of functional protein by GM L. lactis. The L. lactis strains used in this study were genetically modified to secrete human proinsulin (PINS), human IL-10 (hIL10), or PINS together with hIL10 and were designated LL-PINS, LL-hIL10, LL-PINS+hIL10, respectively (Supplemental Figure 1A; supplemental material available online with this article; doi:10.1172/JCI60530DS1). Western blot analysis using specific Abs to PINS and hIL10 revealed the secretion of PINS and hIL10 in the culture supernatant of the respective
L. lactis strains (Supplemental Figure 1B). ELISA further confirmed that PINS was secreted at approximately $2 \mathrm{ng} / \mathrm{ml}$ by LL-PINS and LL-PINS+hIL10 (Supplemental Figure 1C), and hIL10 was secreted at approximately $15 \mathrm{ng} / \mathrm{ml}$ by LL-hIL10 and LL-PINS+hIL10 (Supplemental Figure 1D).

Combination therapy with mucosal delivery of PINS and bIL10 by GM L. lactis and systemic low-dose anti-CD3 stably reverts newly diagnosed diabetes in NOD mice. We first performed a dose titration of anti-CD3 mAb (clone 145-2C11). As previously shown (21), anti-CD3 mono-treatment acted in a dose-dependent manner (Supplemental Figure 2, $\mathrm{A}$ and B). Thus, 4 consecutive daily doses of $2.5,5$, and $10 \mu \mathrm{g}$ anti$\mathrm{CD} 3 \mathrm{mAb}$ reverted T1D in $25 \%(n=32), 33 \%(n=3)$, and $50 \%(n=4)$ of the mice, respectively. Interestingly, a further increase in the antiCD3 dosage resulted in a progressive loss of treatment efficacy, and doses of $50 \mu \mathrm{g}$ even resulted in acute toxicity with weight loss, diarrhea, and death (data not shown and Supplemental Figure 2B).

Next, we tested whether mucosal vaccination using L. lactis secreting PINS along with hIL10 (LL-PINS+hIL10) could restore normoglycemia after diabetes onset. For this, newly diagnosed diabetic NOD mice received oral doses of LL-PINS+hIL10 $\left(2 \times 10^{9} \mathrm{CFU}\right.$, 5 days per week for 6 weeks) with or without an initial short course of systemic, subtherapeutic doses of anti-CD3 mAb (2.5 $\mu \mathrm{g}$, days 0-4) (see regimen in Supplemental Figure 3A). 
A
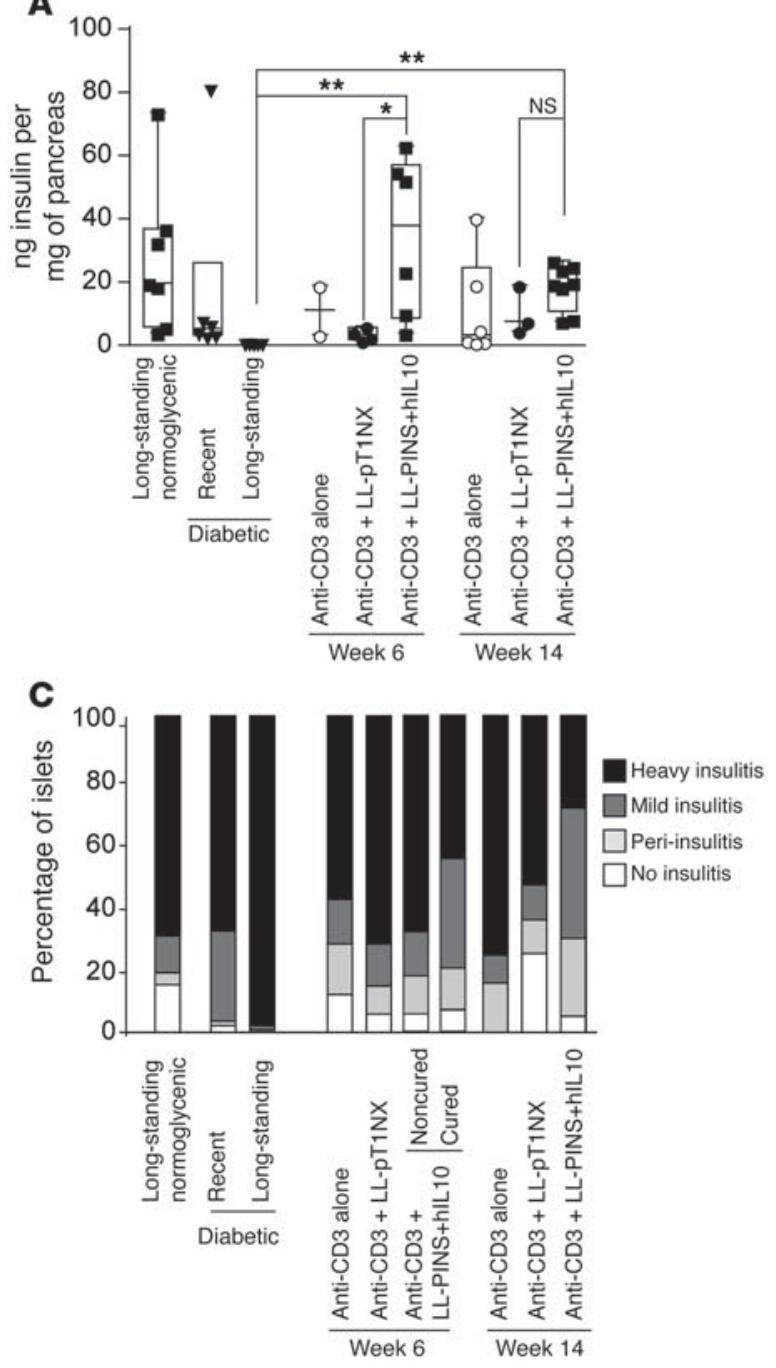

B Long-standing
normoglycemic
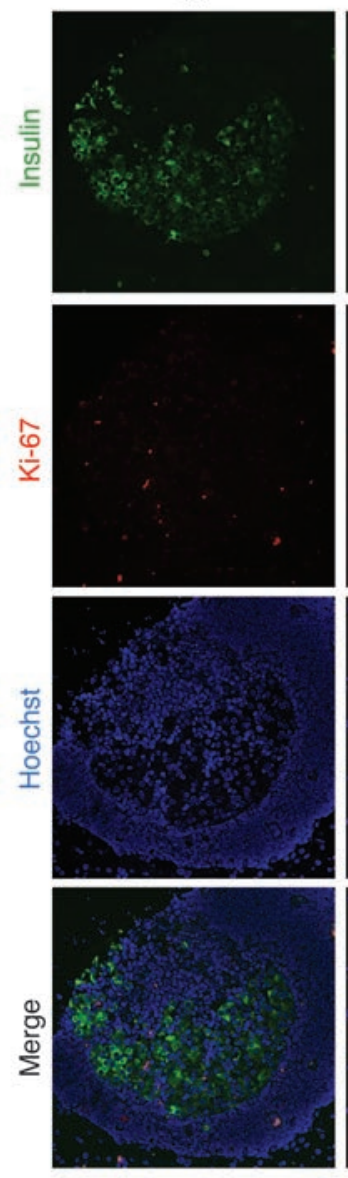
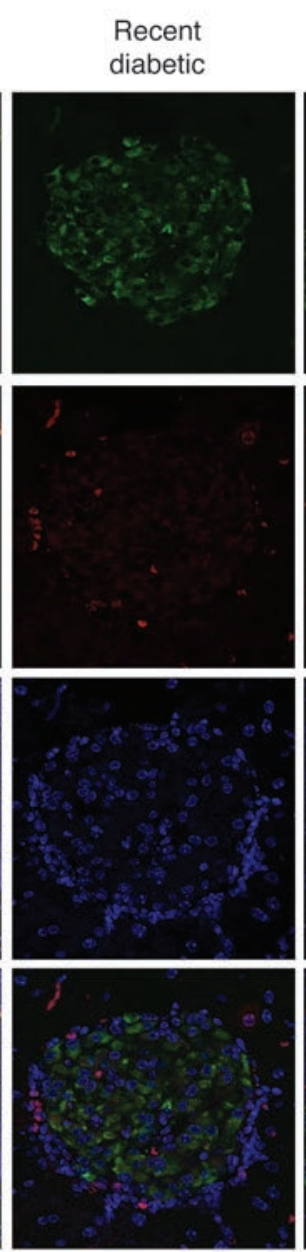

Anti-CD3 + LL-PINS+hIL10
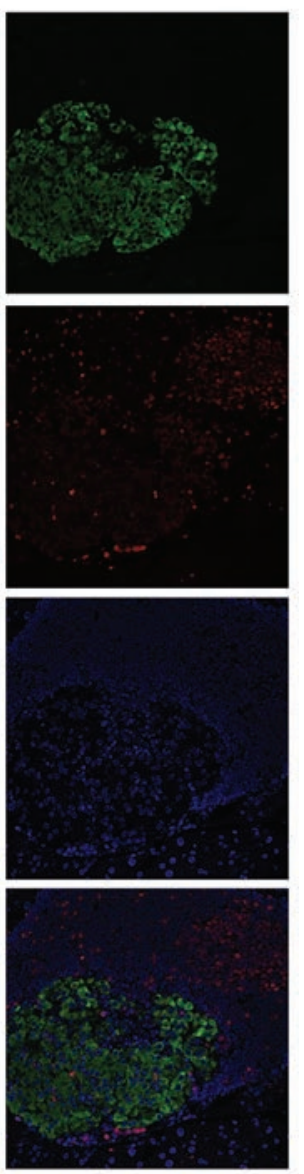

\section{Figure 2}

CT preserves insulin content and limits insulitis. (A) Evaluation by ELISA of insulin content in pancreatic extracts from cured (14 weeks) and control experimental groups, as indicated. Data are expressed as $\mathrm{ng} / \mathrm{mg}$ of pancreas. Shown are the individual values (symbols) overlaid with mean (horizontal line), box (25th and 75th percentile), and Tukey whiskers. Statistical significance between 2 groups was calculated using Mann-Whitney $U$ test; ${ }^{*} P<0.05,{ }^{* \star} P<0.01$. (B) Pancreatic sections were stained for insulin (green), Ki-67 (red), and DNA by Hoechst (blue) and analyzed with confocal microscopy at $\times 63$ original magnification. Representative Ki-67/insulin microphotographs documenting the absence of proliferating $\beta$ cells in the pancreas are shown. (C) Pancreatic sections of the indicated groups were stained with H\&E, and insulitis was scored as indicated.

As expected, untreated control animals remained hyperglycemic (9 of 9 mice) and were euthanized (mean survival, $36.4 \pm 4.9$ days) when they had lost more than $20 \%$ of their initial body weight (Figure 1A). Mucosal delivery of PINS and hIL10 by LL-PINS+hIL10 cured diabetes in $15 \%$ of treated animals, and this could be readily observed up until 100 days after treatment (Figure 1A). Low-dose anti-CD3 alone or in combination with the empty vector control strain LL-pT1NX was similarly partially effective in reverting diabetes (25\% and $23 \%$ respectively). Strikingly, treatment with LL-PINS+hIL10 in combination with low-dose systemic anti-CD3 $\mathrm{mAb}$ surpassed this level of therapeutic efficacy and stably reverted overt autoimmune diabetes in 59\% of the animals (36 of 61 mice) (Figure 1A), to a greater extent than the combination therapy (CT) with anti-CD3 plus LL-hIL10 or LL-PINS (40\% and 49\% reversal, respectively) (Figure 1A). These treatment regimens were safe and well tolerated: treated animals showed no signs of weight loss or intestinal inflammation or corresponding cytokine production, except for a small increase in IFN- $\gamma$ (Supplemental Figure 3, B-D). Furthermore, compared with anti-CD3 therapy alone, anti-CD3 and LL-PINS+hIL10 CT reverted diabetes faster (within $11.7 \pm 1.2$ vs. $17.6 \pm 3.6$ days) (Supplemental Figure 4), and in the cured mice glycemia levels remained stably normal during the entire observation period rather than continuously oscillating, a feature observed in mice cured by low-dose anti-CD3 therapy (Figure 1B). Of note, mice were followed for more than 14 weeks after treatment initiation, and once normoglycemia was achieved, no recurrence of disease was observed (Figure 1B). The efficacy of therapy was related to the level of residual functional $\beta$ cell mass at the point of treatment initiation (Figure 1C), but the CT not only cured $74 \%$ of mice starting with glycemia of less than $350 \mathrm{mg} / \mathrm{dl}$, but also $33 \%$ of mice with 

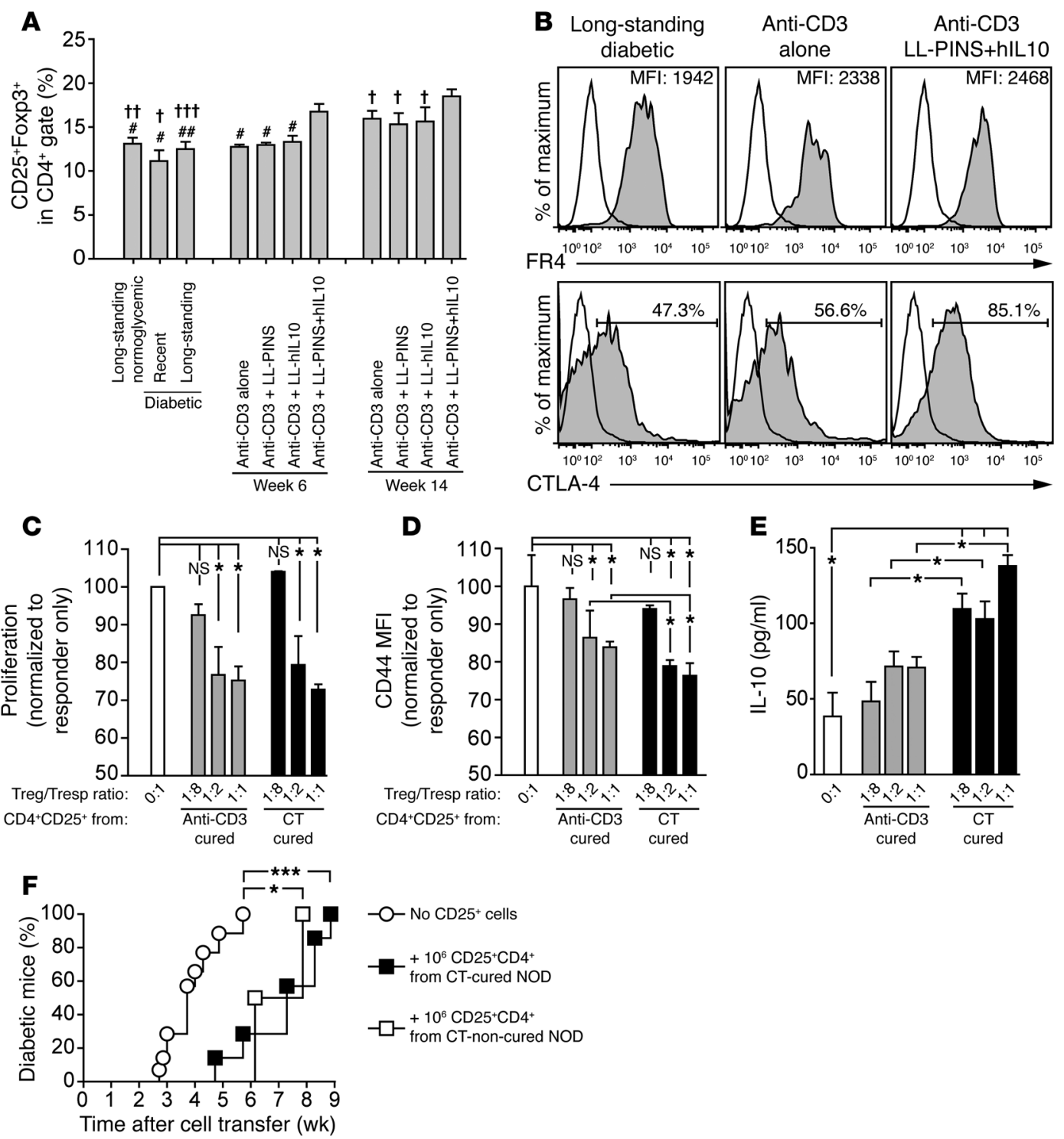

Figure 3

CT increases functional Tregs. (A) CD25+Foxp3 ${ }^{+}$cells in PLNs, shown as mean \pm SEM within the CD4 gate. (B) Histogram overlays of FR4 (top) and CTLA4 (bottom) by CD4+CD25+Foxp3 ${ }^{+}$cells isolated from PLNs (end of treatment). Inset values: MFI of FR4 expression and percentage of CTLA4+ cells in CD4+CD25+Foxp3+ gate, respectively. Gray histograms: staining on indicated sample; solid lines: isotype staining on nondiabetic control sample. (C-E) In vitro polyclonal suppressor assay. CD4+CD25- Tresps isolated from normoglycemic NOD mice were dye labeled and stimulated using $0.5 \mu \mathrm{g} / \mathrm{ml}$ soluble anti-CD3 and accessory cells for 72 hours in the presence of CD4+CD25+ Tregs isolated from cured mice at the end of the indicated treatment. Results of assays for in vitro suppressive capacity are shown. (C) Proliferation of Tresps, shown as percentage of Tresps that had undergone 2 or more divisions, normalized to Tresp-only culture. (D) Activation of Tresps, shown as MFI of CD44 expression, normalized to Tresp-only culture. (E) ELISA for IL-10. (F) Assay for in vivo suppressive capacity. CD25-depleted splenocytes from diabetic NOD mice were transferred into NOD/SCID mice without (white circles) or with CD4+CD25+ cells from CT-cured (white squares)

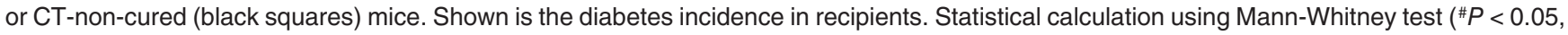
${ }^{\#} P<0.01$ versus week $6 \mathrm{CT} ;{ }^{\dagger} P<0.05,{ }^{\mathrm{t}} P<0.01,{ }^{\mathrm{t} t} P<0.001$ versus week $\left.14 \mathrm{CT}\right), t$ test $(\mathbf{C}-\mathbf{E})$, and Mantel-Cox log-rank (F). Results in $\mathbf{C}-\mathbf{E}$ are representative of at least 3 experiments (each at least 3 treated mice). ${ }^{\star} P<0.05,{ }^{* \star *} P<0.001$.

a starting glycemia greater than $350 \mathrm{mg} / \mathrm{dl}$, in contrast to $40 \%$ and $11 \%$ of mice treated with the low-dose anti-CD3.

$C T$ preserves functional $\beta$ cell mass and reduces severe insulitis. Normoglycemia achieved by CT suggests a preservation of the remaining $\beta$ cell mass as a result of reduced autoimmune destruction and/or increased restoration of pancreatic $\beta$ cells. We found that insulin contents of pancreata from CT-cured animals were restored to the levels present in normoglycemic NOD mice (Figure 2A). Also, pancreata of CT-cured animals contained more insulin than pancreata of untreated diabetic mice or mice treated with anti-CD3 and L. lactis empty vector control (LL-pT1NX), both at the end of the treatment regimen (week 6) and long-term (14 weeks). Morphometric analysis of insulin-positive areas in the islets of CT-cured animals confirmed higher $\beta$ cell content and a preservation of greater num- 

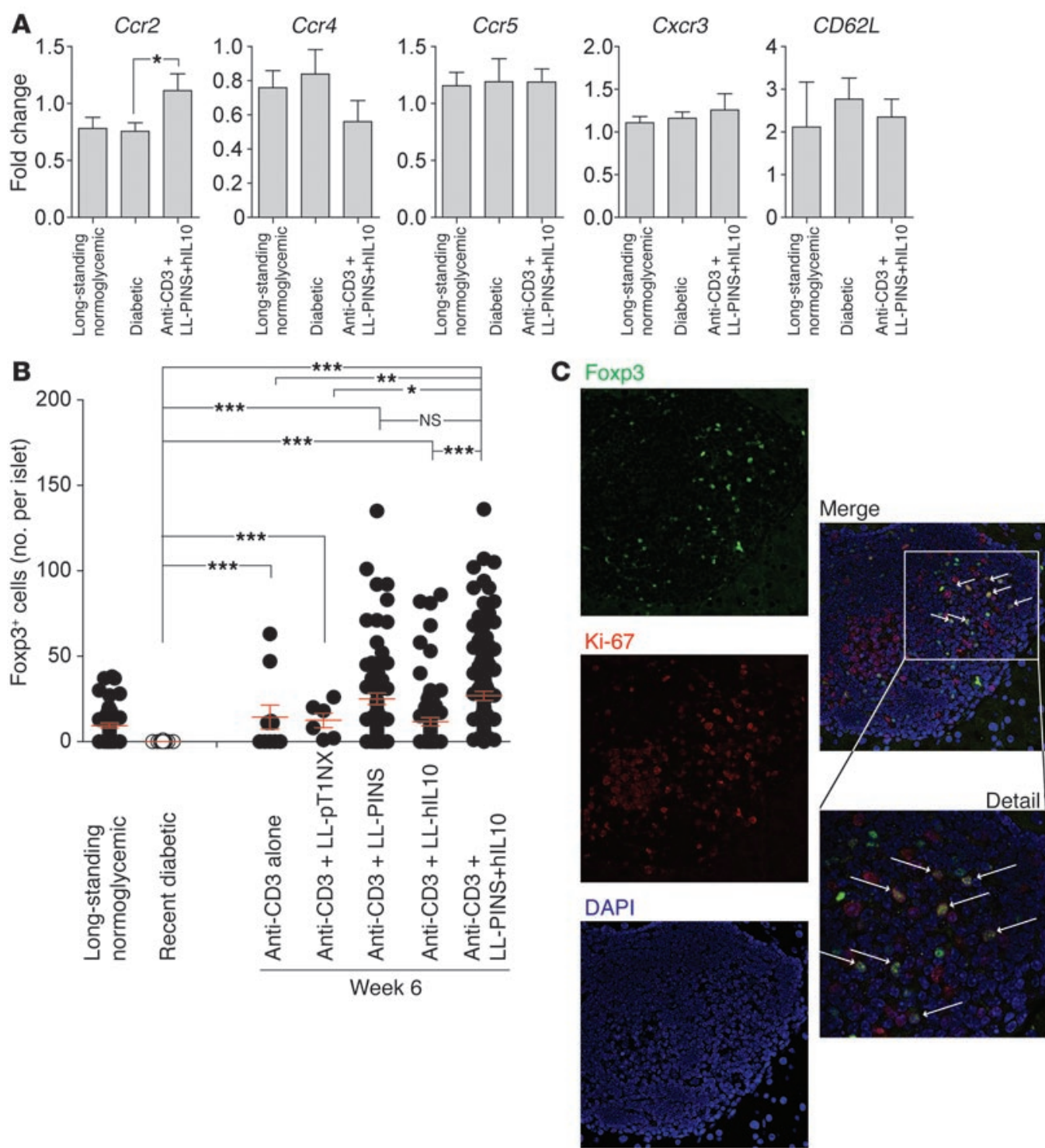

\section{Figure 4}

Local accumulation and autoAg-specific suppression by Tregs upon CT. (A) Real-time PCR analysis of indicated mRNA isolated from CD4+CD25+ T cells sorted from spleen and PLN of long-standing normoglycemic, diabetic, and CT-cured NOD mice. (B) Number of Foxp3-expressing cells in or around the pancreatic islets as determined by manual counting of Foxp $3^{+}$cells on immunostained cryosections as shown in Supplemental Figure 9. (C) Representative microphotographs of immunostaining for Ki-67 and Foxp3 and nuclear staining using DAPI on paraffin sections of the pancreas of CT-cured NOD mice. White arrows indicate Ki-67+Foxp3 ${ }^{+}$double-positive cells, indicating proliferating Foxp3 ${ }^{+}$Tregs. Original magnification, $\times 63$; detail, $\times 252$. Statistical significance was calculated using Mann-Whitney $U$ test $\left({ }^{\star} P<0.05,{ }^{* \star} P<0.01,{ }^{\star \star \star} P<0.001\right)$.

bers of islets (data not shown). Nevertheless, the serum levels of random C-peptide and insulin auto-Abs (IAAs) were comparable in treated versus untreated mice (Supplemental Figure 5 and data not shown). Immunohistological analysis of pancreatic sections from CT-cured animals revealed no proliferating functional $\beta$ cells, as determined by staining for insulin and $\mathrm{Ki}-67$, characteristic markers for $\beta$ cells and proliferating cells, respectively (Figure $2 \mathrm{~B}$ ).

Treatment efficacy was reflected not only in $\beta$ cell parameters, but also in the degree of insulitis. Whereas insulitis worsened in untreated animals, severe insulitis resolved in the majority of CTcured mice $(50.4 \% \pm 10.4 \%$ at 6 weeks and $29.1 \% \pm 4.1 \%$ at 14 weeks after treatment initiation) (Figure 2C).
In conclusion, diabetes reversal by $\mathrm{CT}$ is associated with preservation, but not proliferation, of functional $\beta$ cell mass and a reduction of immune infiltration.

Intact $T$ cell responsiveness upon $C T$. Generalized immunosuppression is an undesirable side effect of many immunotherapies. In this regard, it is known that anti-CD3 treatment can temporarily deplete T cells and shed TCR complexes (22). We confirmed that low-dose anti-CD3 treatment caused temporary depletion of $\mathrm{CD} 4^{+} \mathrm{T}$ cells and TCR shedding on $\mathrm{CD} 44^{\mathrm{lo}} \mathrm{CD} 62 \mathrm{~L}^{\mathrm{hi}} \mathrm{CD} 8^{+} \mathrm{T}$ cells, consequently raising the $\mathrm{CD}^{+} / \mathrm{CD}^{+}$ratio (data not shown). Compared with no treatment, both anti-CD3 monotherapy and CT transiently raised the $\mathrm{CD}^{+} / \mathrm{CD}^{+}$ratio (Supplemental Figure $6 \mathrm{~A}$ ) 
A

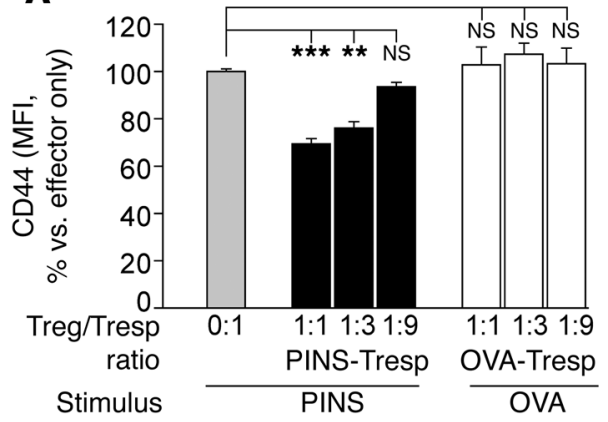

C

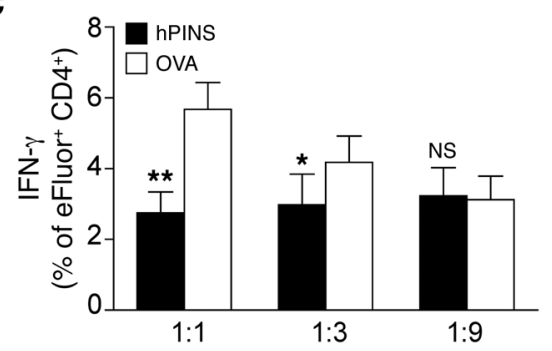

B

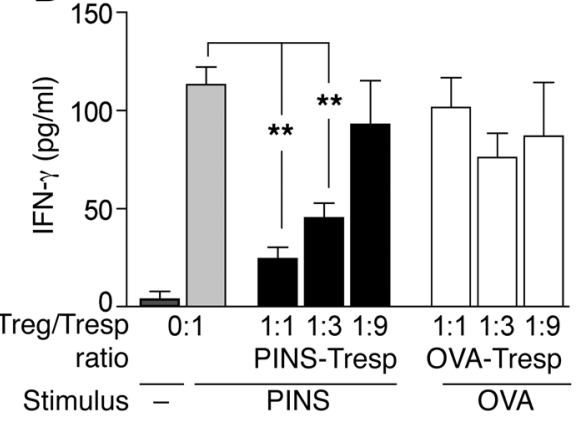

D

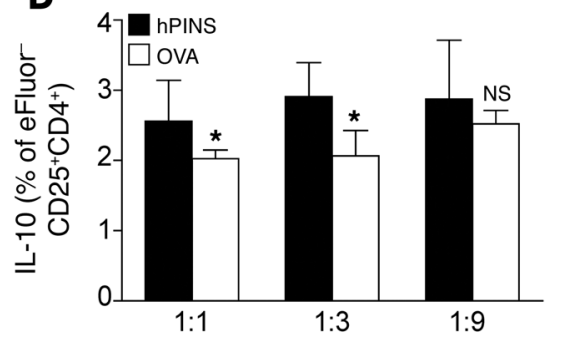

FR4 compared with those in untreated diabetic animals (Figure 3B). Importantly, more $\mathrm{CD} 4^{+} \mathrm{CD} 25^{+} \mathrm{Foxp}^{+}$ Tregs expressed the negative co-stimulatory molecule CTLA-4 in PLNs of CT-treated mice than in those of diabetic controls and anti-CD3-treated animals (Figure 3B).

We next assessed whether the Tregs from CT-cured animals featured enhanced functional capacity. In an in vitro suppression assay, $\mathrm{CD} 4^{+} \mathrm{CD} 25^{+} \mathrm{T}$ cells from CT- or anti-CD3-cured animals similarly counteracted the proliferation of polyclonally stimulated $\mathrm{CD} 4{ }^{+} \mathrm{CD} 25^{-}$responder $\mathrm{T}$ cells (Tresps) from normoglycemic NOD mice (Figure $3 \mathrm{C}$ ). However, $\mathrm{CD} 4^{+} \mathrm{CD} 25^{+} \mathrm{T}$ cells from CT-cured animals not only limited the upregulation of CD69 on Tresps (data not shown), but also restrained the CD44 expression more efficaciously than $\mathrm{CD} 4{ }^{+} \mathrm{CD} 25^{+} \mathrm{T}$ cells from antiCD3-cured mice (Figure 3D), indicating an enhanced capacity to suppress Tresp activation. Interestingly, higher concentrations of the immunomodulatory cytokine IL-10 were detected in the supernatants of cultures containing Tregs from CT-cured mice than from anti-CD3-cured mice (Figure 3E). Comparing Tregs from CT-cured and CT-non-cured NOD mice revealed similar suppression of the proliferation of Tresps in vitro (Supplemental

but did not alter CD44 expression on either $\mathrm{CD}^{+}$and $\mathrm{CD} 8^{+} \mathrm{T}$ cells (data not shown). CT-cured animals did not suffer general immune suppression, because splenic $\mathrm{T}$ cells recovered from CTcured mice responded similarly to those from normoglycemic NOD mice to in vitro stimulation with pharmacological agents or alloAgs (Supplemental Figure 6, B and C). Moreover, CT-cured NOD mice rejected allogeneic (C57BL/6) skin transplants similarly to untreated mice (Supplemental Figure 6D). Taken together, the results indicate that $\mathrm{CT}$ preserves normal $\mathrm{T}$ cell responsiveness to disease-unrelated Ags.

CT increases functional $C D 4^{+} C D 25^{+}$Foxp $3^{+}$Tregs. We next assessed the presence of Tregs by flow cytometry. At both 6 and 14 weeks after treatment initiation, pancreatic lymph nodes (PLNs) of CT-cured mice contained higher frequencies of $\mathrm{CD}^{4}{ }^{+} \mathrm{CD} 25^{+} \mathrm{Foxp}^{+}$Tregs, compared with newly diagnosed diabetics as well as long-standing diabetics or normoglycemic NOD mice (Figure 3A). Moreover, at both time points, CT with anti-CD3 plus LL-PINS+hIL10 was more effective than treatment with anti-CD3 plus LL-PINS or LL-hIL10, indicating the advantage of mucosal co-delivery of PINS and hIL10 in our CT therapy for the induction of long-term tolerance. Similarly, the spleens of CT-cured NOD mice contained more Tregs than those of NOD mice treated with anti-CD3 alone or anti-CD3 plus LL-PINS or LL-hIL10 (Supplemental Figure 7). All $\mathrm{CD}^{+}{ }^{+} \mathrm{CD} 25^{+} \mathrm{Foxp}^{+}$Tregs expressed folate receptor 4 (FR4) (23), but $\mathrm{CT}$ and anti-CD3 monotherapy increased the expression levels of
Figure 8A), but Tregs from CT-cured mice more strongly suppressed IFN- $\gamma$ production by Tresps and contained more IL-10-producing cells than those from CT-non-cured NOD mice (Supplemental Figure 8, B and C). This experiment also indicated that the cellular source of IL-10 in the coculture was the cell proliferation dye-negative $\mathrm{CD} 4^{+} \mathrm{CD} 25^{+}$Treg population (Supplemental Figure $8 \mathrm{C}$ ).

Finally, we evaluated whether Tregs from CT-cured mice transferred disease-specific protection in vivo. For this purpose, NOD/ SCID mice were made diabetic by adoptive transfer of CD25-depleted splenocytes isolated from overtly diabetic female NOD mice $\left(10 \times 10^{6}\right.$ cells). Cotransfer of $1 \times 10^{6} \mathrm{CD} 4^{+} \mathrm{CD} 25^{+} \mathrm{T}$ cells from spleens and PLNs from CT-cured mice significantly delayed the onset of diabetes (median onset, 26 vs. 50 days; Figure 3F). Interestingly, transferring equal cell numbers of $\mathrm{CD} 4^{+} \mathrm{CD} 25^{+} \mathrm{T}$ cells from CT-non-cured NOD mice similarly delayed diabetes onset, indicating that the CT also increased the numbers of functional Tregs in CT-non-cured animals, albeit to a lesser extent that in CT-cured mice.

Taken together, the results indicate that CT with low-dose antiCD3 and LL-PINS+hIL10 increases the frequency of functional Tregs that suppress polyclonally in vitro and disease specifically in vivo.

Tregs accumulate and proliferate in the pancreatic islets upon CT. Targeting the proper location is important for Tregs to exert their maximum potential. We first measured the mRNA levels of molecules that can control trafficking to sites of inflammation, such as $\mathrm{Ccr} 2, \mathrm{Ccr} 4, \mathrm{Crr} 5, \mathrm{Cxcr} 3$, and $C D 62 \mathrm{~L}$. We found that, compared with 
$\mathrm{CD} 4^{+} \mathrm{CD} 25^{+} \mathrm{T}$ cells sorted from long-standing normoglycemic and diabetic controls, in $\mathrm{CD} 4^{+} \mathrm{CD} 25^{+} \mathrm{T}$ cells from CT-treated animals there were increased copy numbers of Ccr 2 (about $50 \%$ more) and, to a lesser extent, of Cxcr3, but not of Ccr4, Ccr5, or CD62L (Figure 4A), suggesting an increased migration capacity to the site of inflammation, possibly in the pancreatic islets, although this has not been tested directly. Confirming this suggestion, quantification in pancreas cryosections revealed that the numbers of Foxp $3^{+} \mathrm{T}$ cells present in the inflammatory infiltrates cuffing the islets were at least 3 times higher upon CT $\left(43 \pm 6.7\right.$ Foxp $^{+}$cells per islet) than upon anti-CD3 treatment $\left(14.4 \pm 7.1\right.$ Foxp $^{+}$cells per islet) and especially compared with untreated diabetic controls $\left(0.05 \pm 0.05\right.$ Foxp $^{+}$cells per islet) (Figure 4B and Supplemental Figure 9). CT-cured pancreata also contained more Foxp $3^{+}$cells than pancreata from mice cured by anti-CD3 plus LL-hIL10, but not anti-CD3 plus LL-PINS. Interestingly, pancreata from cured mice contained more Foxp $3^{+}$cells than pancreata from non-cured mice, suggesting that the infiltrating Tregs contributed to the remission (Supplemental Figure 10). Strikingly, these Foxp $3^{+}$cells not only remained present in the islets of CT-cured animals as long as 14 weeks after treatment initiation (Supplemental Figure 10), but were also expanding locally, as evidenced by the coexpression of Ki-67 and Foxp3 (Figure 4C).

These data support the idea that CT with LL-PINS+hIL10 and low-dose anti-CD3 increases Treg numbers in the local lymphoid organ (PLN) and causes these Tregs to migrate to and expand in the site of inflammation, where regulation is needed.

CT induces PINS-specific Tregs. AutoAg-specific tolerance is a highly desired feature for safe autoimmune therapy. We assessed whether $\mathrm{CD} 4{ }^{+} \mathrm{CD} 25^{+}$Tregs from CT-cured mice respond specifically to the autoAg PINS and not to an irrelevant Ag OVA. For this, CD4 ${ }^{+}$ Tresps were isolated from the draining lymph nodes of PINS- or OVA-immunized NOD mice (PINS-Tresps and OVA-Tresps, respectively), dye labeled, and restimulated in vitro by activated APCs, previously pulsed with whole PINS or OVA, respectively. Addition of $\mathrm{CD} 4^{+} \mathrm{CD} 25^{+}$Tregs from CT-cured mice significantly prevented the activation of PINS-Tresps, as evidenced by the reduced levels of $\mathrm{CD} 44$ and IFN- $\gamma$ produced by the Tresp population (Figure 5, A-C). These phenomena were PINS specific, as $\mathrm{CD} 4{ }^{+} \mathrm{CD} 25^{+}$Tregs from CT-cured mice did not alter the elevated levels of CD44 and IFN- $\gamma$ in the Tresp population after prime-recall with OVA. Interestingly, Tregs from CT-cured mice also produced more IL-10 in response to PINS antigen (Figure 5D). These data indicate that the Tregs induced by CT with LL-PINS+hIL10 and low-dose anti-CD3 only become functional in the presence of the targeted autoAg, PINS.

\section{Discussion}

Intervention after onset of human T1D using a clinically acceptable dose of a single systemic immunomodulatory compound, such as anti-CD3 mAb in the Protégé study (teplizumab) (10) and the DEFEND-1 study (otelixizumab) (9), or an Ag-specific monotherapy (e.g., GAD65 immunization; ref. 13) has not lived up to the expectations raised by the preclinical successes in animal models or the initial phase II studies $(6,7,24)$. For several years now, preclinical research evidence has prompted experts to propose the combination of systemic immune modulation and Ag-specific interventions as the best therapy to revert T1D $(1,2,25,26)$.

In this study, a novel Ag-based vaccination approach was found to reverse autoimmune diabetes in mice. GM L. lactis bacteria target PINS and a biologically active immunomodulatory cytokine,
IL-10, to the intestinal mucosa and, in combination with low doses of anti-CD3 $\mathrm{mAb}$, reset the immune system toward $\beta$ cell-specific tolerance. Such a therapeutic approach is expected to boost the induction of autoAg-specific immune tolerance for several reasons. First, the gastrointestinal tract is the preferred and most attractive route for the safe delivery of Ags aimed at inducing and/or restoring immune tolerance. Second, in the gut, IL-10 especially can act locally on intestinal epithelial cells, thereby enhancing Ag capture, and on APCs by influencing Ag presentation through modulation of MHC class II. This can lead to preferential priming of naive $\mathrm{T}$ cells to generate Tregs and/or directly act on Tregs to maintain Foxp3 expression and their suppressive function $(27,28)$. Third, the observations by Peakman's group suggest that misfolded and misprocessed PINS-derived peptides are crucial and early autoAgs in T1D (29). Fourth, anti-CD3 mAb treatment induces migration of human Tregs to the intestine (30) and thus promotes Treg interaction with the Ags delivered in the intestine. Additionally, antiCD3 can temporarily and dose-dependently halt C-peptide decline in newly diagnosed patients as demonstrated in the studies using high doses of anti-CD3 mAbs, pointing toward the potential of this intervention in altering the course of human T1D $(5,6)$. However, side effects, although manageable, remain a concern. There is thus a need for other strategies, including short-term application of lower doses of anti-CD3 mAb such as in the CT presented here.

Our intervention effectively reverted diabetes in newly diagnosed diabetic NOD mice, with a success rate seldom approached in NOD mice. The therapy allowed us to exploit lower doses of antiCD3 (ideally to circumvent side effects and undesired reactions) in combination with other interventions to enhance therapeutic efficacy. In particular, our CT using L. lactis expressing PINS and hIL10 and low-dose anti-CD3 preserved functional $\beta$ cell mass, resolved severe insulitis, and increased the frequencies of functional CD $4^{+} \mathrm{CD} 25^{+} \mathrm{Foxp}_{3}{ }^{+} \mathrm{CTLA} 4^{+}$Tregs. In vitro, Tregs from CT-cured mice were more potent than Tregs from anti-CD3-cured mice in suppressing $\mathrm{CD}^{+} \mathrm{T}$ cell responses. Tregs from CT-treated mice also transferred protection in vivo, in line with the concept that PINS is indeed a crucial autoAg (31-33). Nevertheless, the observed production of IL-10 by these Tregs in an in vitro suppression assay and the stable reversal of diabetes suggest bystander suppression. We further demonstrated that these Tregs accumulate in draining PLNs and pancreatic islets and respond in an Ag-specific manner as evidenced by their ability to effectively suppress $\mathrm{T}$ cell responses in the presence of the autoAg PINS. It is conceivable that the Ag-specificity of the Tregs in CT-cured mice contributed to their increased pancreatic accumulation and observed proliferation. Although the results are suggestive, we cannot conclude from our studies whether the relationship between Treg induction and clinical outcome is truly causal or merely associative.

Interestingly, these immune effects, in particular the induction of Ag-specific Tregs, were present independent of whether the treated mice were cured, but subtle differences in numbers and characteristics of Tregs induced by the CT were observed between cured and non-cured mice. Thus, CT-non-cured mice had lower numbers of Tregs in the pancreas, and Tregs from cured mice were more efficient in secreting IL-10 and suppressing IFN- $\gamma$ production by responder $\mathrm{T}$ cells. These small differences suggest that other factors, such as remaining functional $\beta$ cell mass at diagnosis, also contributed to clinical outcome. This mass, as measured by insulin content and histology, increased under therapy and remained higher than at diabetes onset throughout the study. The CT could 
even cure mice with very high glycemic levels (>350 mg/dl) at onset. We did not detect $\beta$ cell proliferation in histological samples, suggesting that therapy caused re-granulation or reactivation of $\beta$ cells silenced by immune inflammation, but could only be clinically effective when a sufficient number of $\beta$ cells were present at treatment initiation. These observations further strengthen the argument that early diagnosis of T1D and the rapid initiation of immunomodulatory therapies will be necessary for efficacy (34).

In 2006, Bresson et al (35) suggested a potential synergy between Ag-specific modalities and anti-CD3 therapy in reverting recentonset T1D. In that study, intranasally administered human PINS along with a 5-day course of systemically administered anti-CD3 $\mathrm{F}\left(\mathrm{ab}^{\prime}\right)_{2}$ fragments reverted recent-onset diabetes in two different experimental T1D models, and, as in our study, therapeutic efficacy was associated with the emergence of PINS-responsive Tregs. Of note, compared with our group, Bresson et al. used higher doses of $\mathrm{Ab}$ but in an Fc-non-binding format. Importantly, the use of free Ag may be extremely time consuming when translated to human disease because of its critical dependence on factors including purity, source, dose, and mode of Ag presentation to the mucosal immune system. Here, active intestinal synthesis and delivery of an Ag by GM L. lactis obviated the need for large-scale purification of human (auto)Ags. Furthermore, in other diseases, oral application of GM L. lactis bacteria repeatedly proved more effective than administration of free purified proteins alone, highlighting not only the efficacy of topical immunomodulation, but also the importance of the mode of therapeutic protein presentation $(36,37)$. Finally, we can state that the outcome of our therapy did not result from the direct exposure to microbial products, known to prevent diabetes in NOD mice (38), because the L. lactis empty vector control LL-pT1NX did not revert diabetes.

The introduction of the GM L. lactis may prove a major step forward in Ag-based tolerance regimens for T1D as it can be rapidly translated to clinical use. L. lactis is a Gram-positive bacterium that has been used since ancient times in preparation of food products, and it is widely recognized as being completely safe for human consumption. When delivered to cells in the gastrointestinal tract, GM L. lactis bacteria reside there for 8-48 hours, during which time they continuously release therapeutic amounts of biologically active proteins. Such a release profile more closely mimics the way in which natural biomolecules are released in the body, in contrast to the pharmacokinetic profile of many conventional drug products. These lactic acid bacteria can be genetically modified to efficiently express any desired protein or peptide without disturbing their biological activity. New prototypes of GM L. lactis strains expressing single as well as multiple human $\beta$ cell-specific $\mathrm{Ag}(\mathrm{s})$ are currently under investigation. Safety is guaranteed by the non-colonizing, noninvasive, and food-grade nature of the bacterium. Also, in terms of deliberate release, an elegant strategy for environmental containment of GM bacteria was developed and positively evaluated by several health authorities and biosafety committees. First-in-human trials have been performed in colitis (39) and are in progress in oral mucositis (ClinicalTrials.gov identifier NCT00938080).

In conclusion, we have demonstrated that orally administered L. lactis modified to concurrently secrete whole human PINS and hIL10 can stably reinstates normoglycemia in newly diagnosed diabetic NOD mice when introduced during the window of opportunity for tolerance created by low-dose systemic anti-CD3 $\mathrm{mAb}$. Combination-treated NOD mice had higher $\beta$ cell mass and augmented levels of functional autoAg-responsive Foxp $3^{+}$Tregs, which resided and proliferated locally in the pancreas. Experience with this bacterial delivery tool in other human diseases and its acceptance by the regulatory authorities should allow rapid translation into the T1D clinical setting.

\section{Methods}

GMLL-PINS+bIL10. The lactococcal vectors used in the present study are listed in Supplemental Figure 1A. Details on their construction, culture, and in vitro quantification and functionality are presented in Supplemental Methods.

Diabetes intervention. Newly diagnosed diabetic NOD mice were treated for 5 consecutive days i.v. (days $0-4 ; 2.5 \mu \mathrm{g} /$ mouse) with hamster anti-mouse CD3 mAb (clone 145-2C11, Bio X Cell). This therapy was given alone or in combination with intragastric inoculation of LL-pT1NX, LL-PINS, LL-hIL10, or LL-PINS+hIL10 $\left(2 \times 10^{9} \mathrm{CFU}\right) 5$ times per week during 6 weeks (Supplemental Figure 3A). Control mice were left untreated. Individual glycemia values at the start of treatment were recorded. Mice were tested 3 times weekly for weight and blood glucose status. Remission was defined as the absence of glycosuria and a return to normal glycemia levels. Treated animals were sacrificed immediately after or 8 weeks after therapy was discontinued, and sera were collected for IAA detection (40) and random C-peptide measurements by ELISA (Millipore) and pancreata were harvested for insulin content determination and/or histological analyses as previously described (see Supplemental Methods). Lymphoid organs (spleen and PLNs) were harvested, and single cells were assessed for phenotyping as described in Supplemental Methods. Detailed methodology and references on Ag-specific suppression and cytokine assays, as well as adoptive transfer experiments, are described in Supplemental Methods.

Islet-associated Foxp $3^{+}$cell detection. Pancreata isolated from cured and noncured mice on different therapies (6 weeks after initiation of treatment) or long-standing normoglycemic or diabetic NOD mice were snap-frozen in 2-methylbutane 99\% (ACROS Organics) and cut into 12- $\mu \mathrm{m}$ tissue sections. Afterward, sections were fixed in $70 \%$ ethanol, dried for 45 minutes, and rehydrated with TBS/proteinase I. Double immunostaining for Foxp3 and insulin was performed. To detect Foxp3, antibodies were applied as follows: FITC-conjugated anti-Foxp3 primary Ab (clone FJK-16a; $5 \mu \mathrm{g} / \mathrm{ml}$ ) from eBioscience was used in TBS/proteinase I with 5\% BSA overnight at $4^{\circ} \mathrm{C}$. Foxp3 signal was first revealed by using Alexa Fluor 488-conjugated rabbit anti-FITC secondary Ab (Molecular Probes, Invitrogen; $12.5 \mu \mathrm{g} / \mathrm{ml}$ ). In order to amplify the signal of Foxp3, an Alexa Fluor 488-conjugated anti-rabbit IgG was used (Molecular Probes, Invitrogen; $8 \mu \mathrm{g} / \mathrm{ml}$ ). Following Foxp3 staining, insulin was detected with guinea pig anti-swine primary Ab from Dako ( $0.3 \mathrm{mg} / \mathrm{ml})$; after 1 hour of incubation, Alexa Fluor 555-conjugated goat anti-guinea pig IgG (Molecular Probes, Invitrogen; $4 \mu \mathrm{g} / \mathrm{ml}$ ) was applied for 1 hour. Proliferation of Foxp $3^{+}$cells was observed by double staining with Ki-67 (as described in Supplemental Methods).

Real-time PCR. We extracted total RNA from sorted $\mathrm{CD} 4^{+} \mathrm{CD} 25^{+} \mathrm{T}$ cells (QIAGEN) isolated from spleen and PLNs from CT-cured as well as longstanding normoglycemic and diabetic NOD mice; subjected it to reverse transcription (Invitrogen); and quantified Ccr2, Ccr4, Ccr5, Cxcr3, and CD62L mRNA by real-time PCR using the StepOne real-time PCR system (Applied Biosystems) as described previously (41).

Statistics. Survival analyses with Kaplan-Meier estimates were used to evaluate the difference in the incidence of diabetes onset between the different groups of NOD mice, with the differences being determined by Mantel-Cox log-rank test analysis. All data are reported as mean \pm SEM. We used 2-tailed Student's $t$ test for group comparison of parametric data or Mann-Whitney rank-sum test for nonparametric data. A $P$ value of less than 0.05 was considered significant in all cases.

Study approval. Animal work in all studies was approved by the Ethical Committee of KUL under project number 185-2009. 


\section{Acknowledgments}

We thank Adrian Liston (VIB Autoimmune Genetics Laboratory, KUL) for constructive reading and comments; Eefje Verdrengh, Jos Depovere, and Jos Laureys for excellent technical assistance; and Tine Paessens, Peter Vanhoenacker, and Annemie Ducatteeuw for help with the L. lactis engineering and ELISA analysis. This work was supported by grants from the Fund for Scientific Research Flanders (FWO-Vlaanderen G.0734.10), KUL (GOA 2009/10), the Belgian government (Interuniversity Attraction Poles, program P6/40 of the Belgian Federal Science Policy Office), the European Community's Health Seventh Framework Programme (FP7/20092014 under grant agreement 241447 with acronym NAIMIT), and from the Juvenile Diabetes Research Foundation (JDRF 17-2011524). T. Takiishi is supported through an SBA fellowship and T.L. Van Belle by an F+ fellowship from KUL. S. Robert is holder of a Baekeland fellowship from the Agency for Innovation by Science and Technology in Flanders (IWT-90702). C. Mathieu is a clinical researcher and $\mathrm{H}$. Korf a postdoctoral fellow of the FWO-Vlaanderen. C. Gysemans is supported by the European Community's Health Seventh Framework Programme (FP7/2009-2014 under grant agreement 241447 with acronym NAIMIT) and KUL. F. Dotta received support from the Italian Ministry of Research, the Italian Ministry of Health, and the Italian Foundation for Diabetes Research (FoRiSID).

Received for publication August 16, 2011, and accepted in revised form February 29, 2012.

Address correspondence to: Chantal Mathieu, Campus Gasthuisberg O\&N1, Clinical and Experimental Endocrinology, Herestraat 49 bus 902, 3000 Leuven, Belgium. Phone: 32.16.346023; Fax: 32.16.330718; E-mail: Chantal.Mathieu@uzleuven.be.
1. Van Belle TL, Coppieters KT, Von Herrath MG Type 1 diabetes: etiology, immunology, and therapeutic strategies. Physiol Rev. 2011;91(1):79-118.

2. Bluestone JA, Herold K, Eisenbarth G. Genetics, pathogenesis and clinical interventions in type 1 diabetes. Nature. 2010;464(7293):1293-1300.

3. Chatenoud L, Thervet E, Primo J, Bach JF. AntiCD3 antibody induces long-term remission of overt autoimmunity in nonobese diabetic mice. Proc Natl Acad Sci U S A. 1994;91(1):123-127.

4. von Herrath MG, Coon B, Wolfe T, Chatenoud L. Nonmitogenic CD3 antibody reverses virally induced (rat insulin promoter-lymphocytic choriomeningitis virus) autoimmune diabetes without impeding viral clearance. J Immunol. 2002; 168(2):933-941.

5. Herold KC, et al. Anti-CD3 monoclonal antibody in new-onset type 1 diabetes mellitus. $N$ Engl J Med. 2002;346(22):1692-1698.

6. Keymeulen B, et al. Insulin needs after CD3-antibody therapy in new-onset type 1 diabetes. $N$ Engl J Med. 2005;352(25):2598-2608.

7. Herold KC, et al. A single course of anti-CD3 monoclonal antibody hOKT3gamma1(Ala-Ala) results in improvement in C-peptide responses and clinical parameters for at least 2 years after onset of type 1 diabetes. Diabetes. 2005;54(6):1763-1769.

8. Keymeulen B, et al. Transient Epstein-Barr virus reactivation in $\mathrm{CD} 3$ monoclonal antibody-treated patients. Blood. 2010;115(6):1145-1155.

9. GlaxoSmithKline and Tolerx announce phase III DEFEND-1 study of otelixizumab in type 1 diabetes did not meet its primary endpoint [press release]. Cambridge, Massachusetts, USA: Associated Press; March 11, 2011.

10. Sherry N, et al. Teplizumab for treatment of type 1 diabetes (Protege study):1-year results from a randomised, placebo-controlled trial. Lancet. 2011; 378(9790):487-497.

11. Daniel D, Wegmann DR. Protection of nonobese diabetic mice from diabetes by intranasal or subcutaneous administration of insulin peptide B-(9-23). Proc Natl Acad Sci U S A. 1996;93(2):956-960.

12. Zhang ZJ, Davidson L, Eisenbarth G, Weiner HL. Suppression of diabetes in nonobese diabetic mice by oral administration of porcine insulin. Proc Natl Acad Sci U S A. 1991;88(22):10252-10256.

13. Wherrett DK, et al. Antigen-based therapy with glutamic acid decarboxylase (GAD) vaccine in patients with recent-onset type 1 diabetes: a randomised double-blind trial. Lancet. 2011;378(9788):319-327.

14. Matthews JB, et al. Developing combination immu- notherapies for type 1 diabetes: recommendations from the ITN-JDRF Type 1 Diabetes Combination Therapy Assessment Group. Clin Exp Immunol. 2010; 160(2):176-184.

15. Steidler L, et al. Treatment of murine colitis by Lactococcus lactis secreting interleukin-10. Science. 2000;289(5483):1352-1355

16. Huibregtse IL, et al. Induction of antigen-specific tolerance by oral administration of Lactococcus lactis delivered immunodominant DQ8-restricted gliadin peptide in sensitized nonobese diabetic Abo Dq8 transgenic mice. J Immunol. 2009;183(4):2390-2396.

17. Rottiers P, De Smedt T, Steidler L. Modulation of gut-associated lymphoid tissue functions with genetically modified Lactococcus lactis. Int Rev Immunol. 2009;28(6):465-486.

18. Steidler L, et al. Biological containment of genetically modified Lactococcus lactis for intestinal delivery of human interleukin 10. Nat Biotechnol. 2003; 21(7):785-789.

19. Steidler L, Rottiers P. Therapeutic drug delivery by genetically modified Lactococcus lactis. Ann NY Acad Sci. 2006;1072:176-186.

20. Steidler L, Rottiers P, Coulie B. Actobiotics as a novel method for cytokine delivery. Ann NY Acad Sci. 2009;1182:135-145.

21. Mehta DS, Christmas RA, Waldmann H, Rosenzweig M. Partial and transient modulation of the CD3-T-cell receptor complex, elicited by low-dose regimens of monoclonal anti-CD3, is sufficient to induce disease remission in non-obese diabetic mice. Immunology. 2010;130(1):103-113.

22. Chatenoud L, Bluestone JA. CD3-specific antibodies: a portal to the treatment of autoimmunity. Nat Rev Immunol. 2007;7(8):622-632.

23. Yamaguchi $\mathrm{T}$, et al. Control of immune responses by antigen-specific regulatory $T$ cells expressing the folate receptor. Immunity. 2007;27(1):145-159.

24. Ludvigsson J, et al. Extended evaluation of the safety and efficacy of GAD treatment of children and adolescents with recent-onset type 1 diabetes: a randomised controlled trial. Diabetologia. 2011;54(3):634-640.

25. Boettler T, von Herrath M. Immunotherapy of type 1 diabetes - how to rationally prioritize combination therapies in T1D. Int Immunopharmacol. 2010;10(12):1491-1495

26. Luo X, Herold KC, Miller SD. Immunotherapy of type 1 diabetes: where are we and where should we be going? Immunity. 2010;32(4):488-499.

27. Seifarth C, Pop S, Liu B, Wong CP, Tisch R. More stringent conditions of plasmid DNA vaccination are required to protect grafted versus endog- enous islets in nonobese diabetic mice. J Immunol. 2003;171(1):469-476

28. Murai M, et al. Interleukin 10 acts on regulatory $\mathrm{T}$ cells to maintain expression of the transcription factor Foxp3 and suppressive function in mice with colitis. Nat Immunol. 2009;10(11):1178-1184.

29. Skowera A, et al. CTLs are targeted to kill beta cells in patients with type 1 diabetes through recognition of a glucose-regulated preproinsulin epitope. J Clin Invest. 2008;118(10):3390-3402.

30. Waldron-Lynch F, et al. Teplizumab treatment induces migration of human $t$ regulatory lymphocytes to the small intestine in vivo. Diabetes. 2011;60(suppl 1):A90.

31. Narendran P, Mannering SI, Harrison LC. Proinsulin-a pathogenic autoantigen in type 1 diabetes. Autoimmun Rev. 2003;2(4):204-210.

32. Peakman $M$, von Herrath $M$. Antigen-specific immunotherapy for type 1 diabetes: maximizing the potential. Diabetes. 2010;59(9):2087-2093.

33. Thrower SL, et al. Proinsulin peptide immunotherapy in type 1 diabetes: report of a firstin-man Phase I safety study. Clin Exp Immunol. 2009;155(2):156-165.

34. Bach JF. Anti-CD3 antibodies for type 1 diabetes: beyond expectations. Lancet. 2011; 378(9790):459-460.

35. Bresson D, et al. Anti-CD3 and nasal proinsulin combination therapy enhances remission from recent-onset autoimmune diabetes by inducing Tregs. J Clin Invest. 2006;116(5):1371-1381.

36. Huibregtse IL, et al. Induction of ovalbumin-specific tolerance by oral administration of Lactococcus lactis secreting ovalbumin. Gastroenterology. 2007;133(2):517-528

37. Vandenbroucke K, et al. Active delivery of trefoil factors by genetically modified Lactococcus lactis prevents and heals acute colitis in mice. Gastroenterology. 2004;127(2):502-513.

38. Wen L, et al. Innate immunity and intestinal microbiota in the development of Type 1 diabetes. Nature. 2008;455(7216):1109-1113.

39. Braat $\mathrm{H}$, et al. A phase I trial with transgenic bacteria expressing interleukin-10 in Crohn's disease. Clin Gastroenterol Hepatol. 2006;4(6):754-759.

40. Wasserfall CH, Atkinson MA. Autoantibody markers for the diagnosis and prediction of type 1 diabetes. Autoimmun Rev. 2006;5(6):424-428.

41. Overbergh L, Giulietti A, Valckx D, Decallonne R, Bouillon R, Mathieu C. The use of real-time reverse transcriptase PCR for the quantification of cytokine gene expression. J Biomol Tech. 2003;14(1):33-43. 